\title{
CARACTERIZACIÓN DE LUMINARIAS TIPO HALURO METÁLICO ANTE EVENTOS SAG
}

\section{Bonie Johana Restrepo CuEstas ${ }^{1}$ HeBer Augusto LÓPEz OSORIO ${ }^{2}$}

\section{RESUMEN}

Este artículo plantea una metodología para la caracterización de luminarias de haluro metálico, ante eventos sag. Inicialmente, se diseñó y construyó un generador de eventos sag. Luego, se planteó un esquema de pruebas, que fue utilizado para analizar el comportamiento de un tipo de luminarias de alta densidad de descarga tipo Metal Halide, ante eventos sag. Finalmente, tomando como referente el estándar SEMI F-47, se realizó la construcción de una curva característica que muestra la región de operación de la luminaria.

PALABRAS CLAVES: Evento Sag; generador sag; luminarias de haluro metálico; pruebas de inmunidad.

\section{CHARACTERIZATION OF METAL HALIDE LAMPS UNDER SAG VOLTAGES}

\section{ABSTRACT}

This paper presents a methodology for characterization of metal halide lamps regarding voltage sags. Initially, a sag generator was designed and implemented. Then, a testing scheme was proposed,in order to analyze the behavior of a type of Metal Halide lamp under this type of event. Finally, taking as reference the standard SEMI F-47, a characteristic curve was constructed, showing the operating region of the lamp.

KEYWORDS: Voltage sag; Sag generator; Metal halide lamps; Immunity test.

\section{CARACTERIZAÇÃO DE LÂMPADAS TIPO HALURO METÁLICO ANTE EVENTOS SAG}

\section{RESUMO}

Este artigo apresenta uma metodologia para a caracterização de lâmpadas de haluros metálicos, ante eventos sag. Inicialmente, foi desenhado e construído um gerador de eventos sag. Logo foi planteado um esquema de provas que foi

1 Ingeniera Eléctrica. Maestría en Ingeniería Eléctrica. Instituto Tecnológico Metropolitano, Medellín- Colombia.

2 Ingeniero Electrónico. Magíster en Gestión Energética Industrial. Instituto Tecnológico Metropolitano, Medellín- Colombia.

Autor de correspondencia: Restrepo Cuestas, B.J. (Bonie

Johana): Carrera 31 \# 54-10 - Parque I, Medellín, Colombia / Tel.; 4600727-5605

Correo electrónico: bonierestrepo@itm.edu.co
Historia del artículo:

Artículo recibido: 13-IV-2016 / Aprobado: 29-XI-2016

Disponible online: 30 de febrero de 2017

Discusión abierta hasta abril de 2018 
usado para analisar o comportamento de um tipo de lâmpadas de alta densidade de cargas tipo Metal Halide ante eventos sag. Finalmente, tomando como referência o padrão SEMI F-47, realizou-se a construção de uma curva característica que mostra a região operacional da lâmpada.

PALAVRAS-CHAVE: Evento sag; Gerador sags; Lâmpadas de haluros metálicos; Provas de imunidade.

\section{INTRODUCCIÓN}

Los eventos sags, también conocidos como dips, son reducciones súbitas, de corta duración, en la magnitud de la señal de tensión eléctrica estándar. Dicha magnitud puede variar entre el 10\% y el 90\% del voltaje (International Electrotechnical Commission, 2006; Markiewicz y Klajn, 2004) ANSI/Atomic Security Agency (ASA). Este tipo de eventos, generalmente se producen por fallas de cortocircuito, o por la puesta en funcionamiento de grandes cargas como motores eléctricos, y pueden afectar el funcionamiento de los dispositivos que compartan circuito de conexión con dichas cargas, incluso con cuando ocurren con una duración inferior a los $100 \mathrm{~ms}$ (Dorr et al., 1995; Yim-Shu Lee et al., 2009; Sedighnejad y Jalilian, 2010).

Los efectos negativos de este tipo de eventos se evidencian principalmente en dispositivos como computadoras, variadores de velocidad, equipos para el control de procesos industriales y luminarias de alta descarga (Pérez Fernández, 2006; Vargas López, 2005). Las luminarias de alta densidad de descarga (high-intensity discharge - HID) más utilizadas en aplicaciones de alumbrado público o lugares de alta afluencia de personas, son las luminarias de alta presión de sodio y las de haluro metálico (Metal Halide). Ambos tipos de luminarias presentan alto nivel de sensibilidad ante la aparición de eventos de corta duración como los sag y swell; estos eventos, que se presentan con regularidad en las redes de distribución eléctrica, ocasionan apagones en las luminarias, con duración entre 5 y 15 minutos mientras se da de nuevo su proceso de ionización para su re-encendido (Díaz et al., 2007; Dorr et al., 1995).

La caracterización del comportamiento de un equipo ante eventos de voltaje del tipo sag, puede realizarse a través de una curva de aceptabilidad de voltaje. Actualmente, los estándares existentes para la obtención de dichas curvas son: CBEMA, ITIC y el Estándar SEMI F-47 (Thallam y Heydt, 2000).

En el presente artículo se presenta una metodología para realizar pruebas de inmunidad ante eventos sag, a luminarias tipo metal halide (haluro metálico). El generador de eventos sag monofásico diseñado y desarrollado para este fin, está basado en la introducción temporizada de una carga inductiva, que ocasiona el hundimiento en la señal de voltaje de alimentación de la luminaria. Se presentan los resultados de la caracterización, usando el generador sag diseñado, para un conjunto de 3 lámparas de haluro metálico de 400W.

\section{ESTRUCTURA DEL GENERADOR DE EVENTOS SAG}

El propósito y función principal del generador de eventos sag es introducir temporalmente una carga de tipo inductivo, que genere una sobrecorriente en la fuente de alimentación. Dicha carga inductiva, provocará una disminución en su señal de tensión, durante un intervalo de tiempo previamente establecido. El generador de eventos sag consta de tres etapas principales que son (Figura 1):

- Etapa de control: que define el inicio de la perturbación y la duración del evento.

- Etapa de conmutación: que permita la conmutación entre el voltaje nominal de alimentación y el voltaje de perturbación.

- Carga inductiva: encargada de provocar el hundimiento de voltaje, al ser introducida temporalmente en el circuito de alimentación de la luminaria. La introducción temporal de dicha carga, se realiza a través de la etapa de conmutación. 
Las características de cada una de las etapas que conforman el sistema, se describen a continuación:

\section{a) Etapa de control:}

De acuerdo con la definición de la norma IEEE 1159, un evento de corta duración instantáneo (tipo sag), es todo hundimiento entre 0,1 y 0,9 p.u., con duración entre 0,5 y 30 ciclos (menos de $500 \mathrm{~ms}$ a $60 \mathrm{~Hz}$ ) (Committee, 2009). Por tanto, el sistema de control se diseñó para definir los tiempos de las interrupciones que se inyectan a alimentación de la luminaria, en el intervalo de operación mencionado. El circuito de control, que debe actuar como un interruptor temporizado, trabaja en un rango de $1 \mathrm{~ms}$ y $800 \mathrm{~ms}$.
Para generar las interrupciones se utilizó un microcontrolador MC68HC08JL3 (MOTOROLA, 2005). El firmware fue desarrollado en leguaje $C$, utilizando como compilador el software CODE-WARRIOR. La operación del circuito de control permite:

Aumentar o disminuir el tiempo de duración del sag, en pasos de $1 \mathrm{~ms}$, para esto son usados pulsadores de ajuste del circuito de control S1 o S4. El tiempo de interrupción seleccionado, se puede visualizar en un display de cristal líquido (LCD, DISP1). Una vez el tiempo ha sido seleccionado, se debe ejecutar la acción, a través S3. Así la interrupción finalizará automáticamente, una vez se haya cumplido el tiempo establecido por el usuario. La Figura 2, muestra el diagrama esquemático y el prototipo del sistema de control.

Figura 1. Estructura del generador de eventos sag

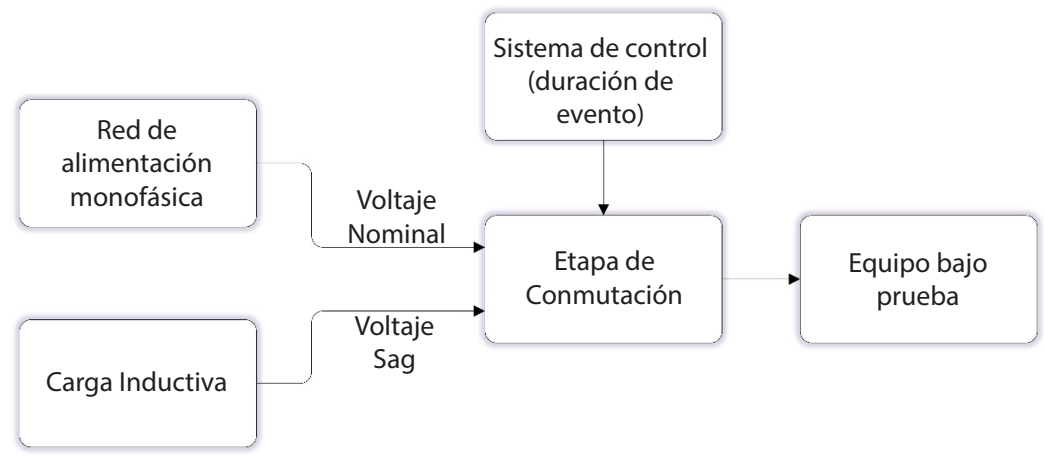

Figura 2. Circuito de control

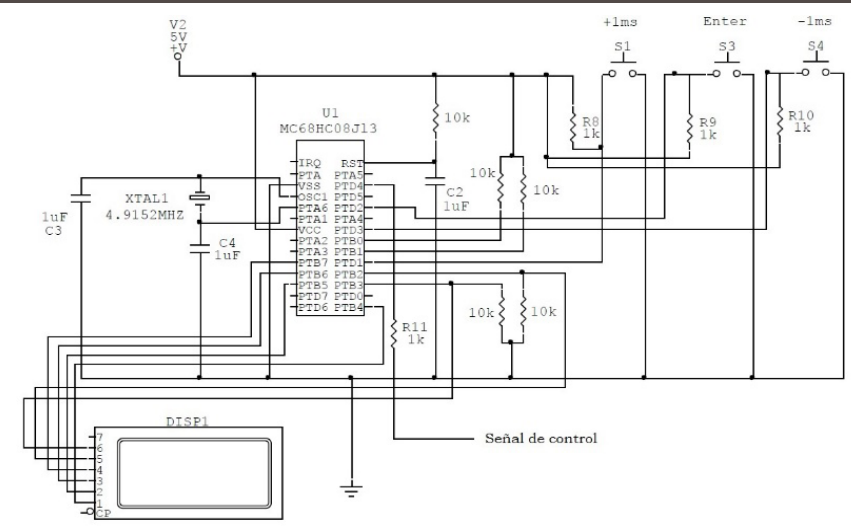

(a) Diagrama esquemático

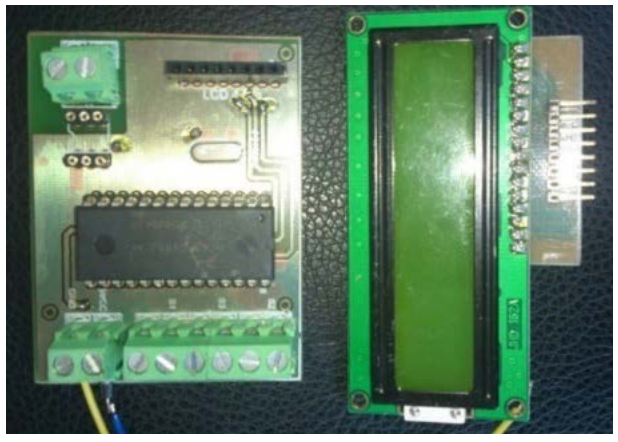

(b) Prototipo 


\section{b) Etapa de conmutación:}

Para realizar la entrada y salida temporal de la carga inductiva que genera el hundimiento en la señal de tensión eléctrica, se utilizó un tiristor de control de fase. Sus características se definen de acuerdo a los valores máximos de corriente en los que puede operar el circuito limitador de corriente de falla. Para este caso se utilizó la referencia ST180S (International Rectifier, 2003). La protección del circuito de conmutación se desarrolló a partir de una red de SNUBBER. El cálculo del condensador SNUBBER se realizó usando el criterio de resonancia (ver Figura 3). Se utilizó un optoacoplador, como interfaz entre la etapa de control y la etapa de potencia.

\section{Figura 3. Circuito de conmutación}

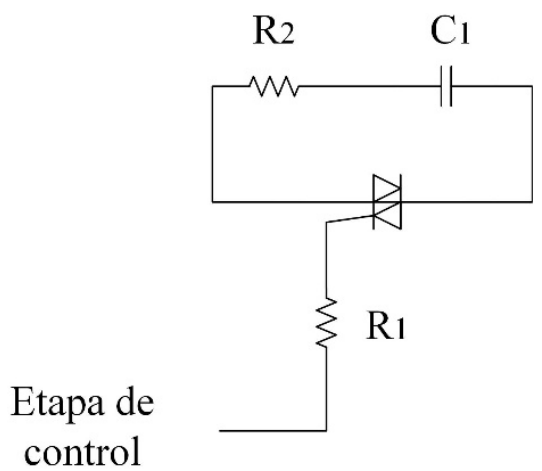

\section{c) Carga inductiva:}

Dado que se requiere una carga inductiva variable que permita realizar un número considerable de evaluaciones, para desarrollar la caracterización del equipo bajo prueba, se seleccionó un variac monofásico. El variac utilizado cuenta con 18 posiciones variables de carga (desde $5 \mathrm{mH}$ hasta $280 \mathrm{mH}$ ) (Ci-Effe-Gi n.d.).

\section{METODOLOGÍA DE PRUEBA}

Para medir el impacto de los sag en un equipo, en este caso luminarias de haluro metálico, es necesario identificar los niveles de hundimiento y duración de los eventos que ocasionan el apagón súbito de la luminaria tipo Metal Halide de 400 W. El método que se planteó en este trabajo se basa en las siguientes etapas:

- Diseño e implementación de un generador de eventos sag, que permita evaluar el comportamiento del equipo (Ver numeral 2).

- Pruebas técnicas y registro de resultados que determinen diferentes niveles de hundimiento y duración de sag, que ocasionen apagones súbitos en la luminaria.

- Curva característica, basada en los niveles y duración de sag que ocasionan apagones súbitos, utilizando las variables empleadas en el estándar SEMI F47.

El montaje para el desarrollo de las pruebas técnicas se puede observar en la Figura 5, donde se presentan los siguientes elementos:

1. Transformador aislador monofásico 110/220V (600W).

2. Balasto electrónico estándar (Referencia ICF2S26H1LD).

3. Equipo bajo prueba: luminaria tipo haluro metálico de 400W.

4. Analizador de calidad de la potencia FLUKE 435 (Fluke Corporation, 2008). Encargado de la medición los eventos generados.

5. Etapa de control de duración del evento.

6. Carga inductiva (variac).

Antes de iniciar las pruebas, es necesario dejar encendida la luminaria durante 30 minutos. Este es el tiempo mínimo requerido para obtener la máxima intensidad.

La prueba se desarrolla seleccionando inicialmente la primera posición de carga del Variac, luego se ajusta en $1 \mathrm{~ms}$ el tiempo de duración de la interrupción del circuito de control. 
Figura 4. Montaje para la realización de las pruebas

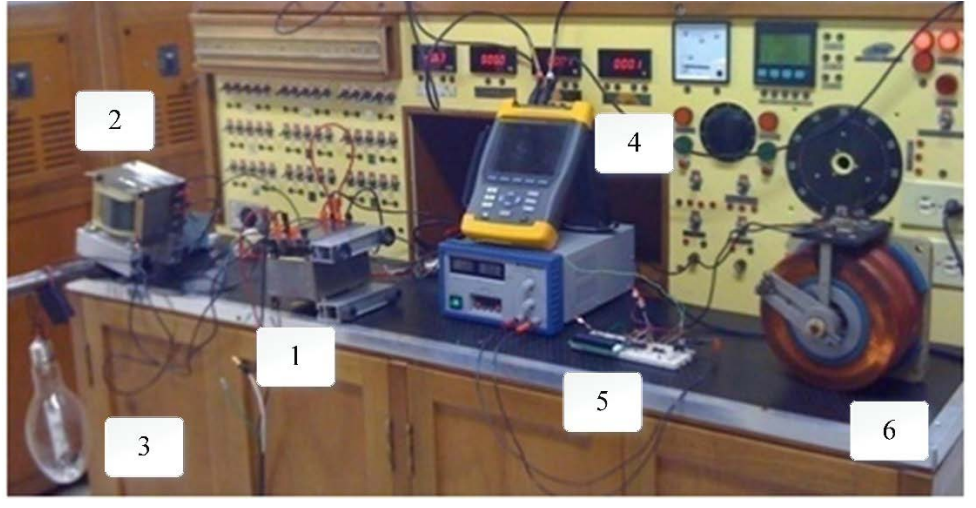

\section{Figura 5. Esquema de pruebas}

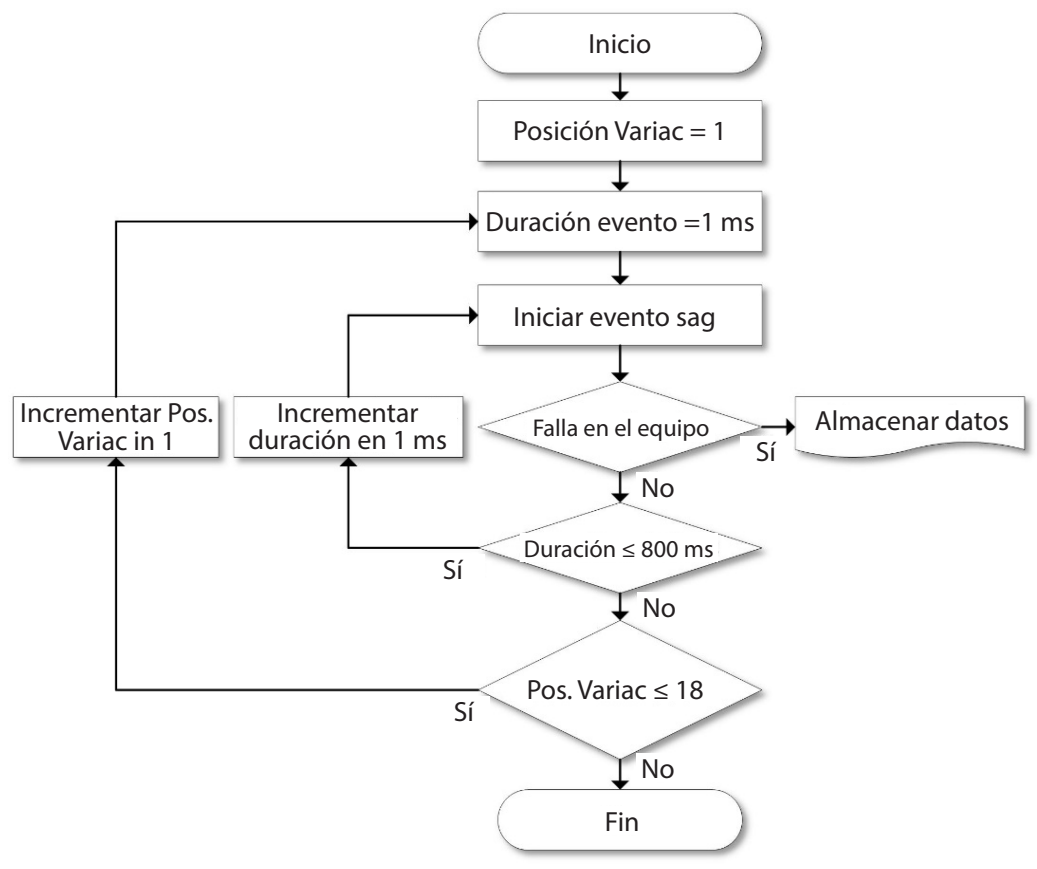

Una vez están dadas estas condiciones, se genera el evento sag desde el sistema de control. El sistema de control envía el comando a la etapa de conmutación, encargada de introducir la carga inductiva. Si la luminaria sufre un apagón, el evento es registrado utilizando el analizador de redes. Si el equipo bajo prueba no falla, se incrementa la duración del evento en $1 \mathrm{~ms}$, y se repite la prueba.

Luego de cada apagón de la luminaria, se esperó un tiempo no inferior a 10 minutos para comenzar una nueva prueba. Esto con el fin de estabilizar su funcionamiento y permitir un restablecimiento de la ionización e intensidad luminosa.

En el caso que no suceda un apagón de la luminaria, es importante el intervalo de tiempo entre muestras sea de la menos 1 minuto. Este tiempo es requerido para lograr una estabilización de la ionización de la luminaria. 
Una vez se ha llegado al límite de duración de eventos del generador, se lleva el variac a la siguiente posición. Esto para modificar el valor de la carga inductiva. Luego se ajusta de nuevo el tiempo de interrupción del circuito de control a $1 \mathrm{~ms}$, para iniciar de esta forma una nueva prueba. El esquema de pruebas se muestra en la Figura 5.

Los datos obtenidos de las 18 posiciones y los diferentes tiempos de duración del evento, permitirán construir la curva de comportamiento de la luminaria ante los eventos sag generados.

\section{RESULTADOS Y DISCUSIÓN}

El esquema de pruebas planteado, se utilizó para evaluar el comportamiento de tres (3) luminarias de alta descarga tipo haluro metálico de 400 W. Para cada luminaria, se realizaron 3 rutinas de pruebas.

Los datos para la construcción de la curva son tomados a partir de la magnitud de la carga configurada desde el Variac, la tensión medida en el momen- to del evento y el tiempo de duración de cada uno de los sag que ocasionaron el apagón de la luminaria.

Para la construcción de la curva característica son considerados los datos de caída de tensión durante cada evento vs. el tiempo de duración del sag, dando cumplimiento a los parámetros establecidos en el estándar SEMI F47 y en la curva CBEMA. Es importante aclarar que el Variac utilizado no presenta un desplazamiento lineal (porcentaje de variación de inductancia).

La Figura 6 muestra la curva característica obtenida. En dicha figura se observa la región de operación para las luminarias analizadas; se tomó como referencia el estándar SEMI F47.

De la Figura 6 se observa que, a mayor duración del evento, la luminaria es más propensa al apagado por causa de la aparición de un sag. Adicionalmente, para cortas duraciones se requiere de una magnitud de hundimiento mucho mayor para que la luminaria se apague.

Figura 6. Curva de caracterización de la luminaria tipo Metal Halide de 400 W

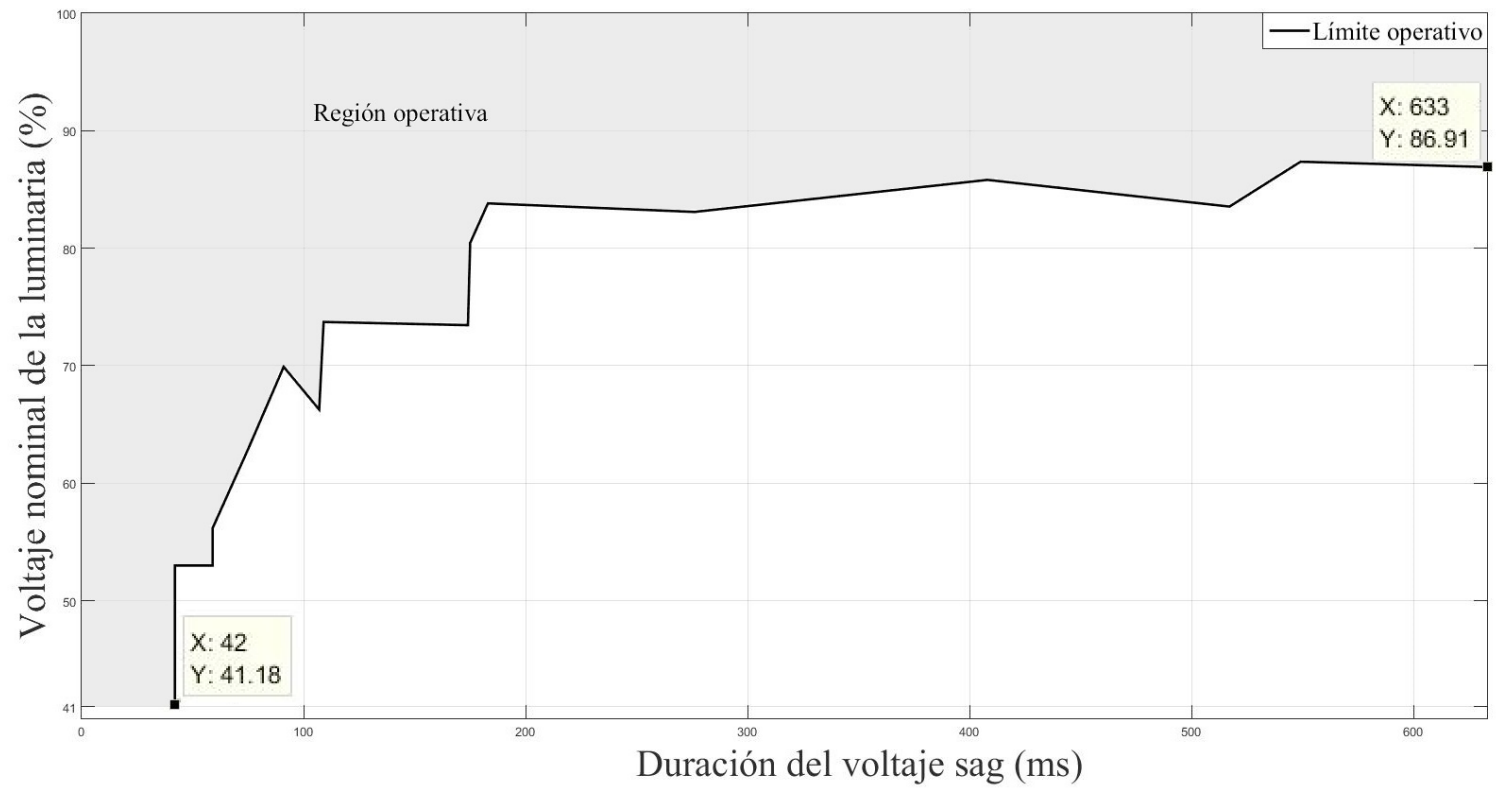


A partir de la curva de caracterización obtenida, se puede establecer la región operativa de las luminarias analizadas (región sombreada). Esta indica que el equipo bajo prueba puede funcionar ante la presencia de sag cuyas características de magnitud y duración estén por encima de la indicada. La región con riesgo de falla, indica que el equipo está expuesto a un apagón y afectará su funcionamiento normal.

Dado el rango de trabajo del variac, no fue posible analizar los eventos sag con duración menor a $42 \mathrm{~ms}$.

Para realizar caracterización de otros tipos de carga con el equipo diseñado, es necesario tener en cuenta la potencia de la red donde está conectada la carga, con el fin de diseñar adecuadamente la fuente requerida.

\section{CONCLUSIONES}

Se implementó un equipo generador de sag simple y de bajo costo, con valores variables tanto en magnitud de voltaje y como en tiempo de duración del evento.

Se planteó una metodología para caracterización de luminarias de haluro metálico, ante eventos sag. La curva utiliza las variables del estándar SEMI F47 (duración del evento vs. el porcentaje de voltaje nominal). El procedimiento de ensayos realizados y la metodología de caracterización planteada en este trabajo es replicable a otros dispositivos eléctricos y electrónicos.

Los datos obtenidos permiten evaluar el comportamiento de una luminaria tipo Metal Halide de $400 \mathrm{~W}$, ante la aparición de eventos sag en la señal de alimentación eléctrica. El análisis realizado a la curva característica construida, permite evidenciar la influencia conjunta entre las variables de duración del evento y su magnitud en la ocurrencia de apagones en el tipo de luminaria analizada.

\section{REFERENCIAS}

Ci-Effe-Gi (S.F.). Variatore monofase da retroquadro a giorno. [Online] Disponible en: http://www.cieffegi. com/prodotti/variatori-monofase-da-retroquadroa-giorno. [Consultado 10 de diciembre de 2015.

Committee, D. (2009). IEEE Std 1159 ${ }^{T M}$-2009, IEEE Recommended Practice for Monitoring Electric Power Quality, Ieee.

Diaz, F.J.; Azcondo, F. J.; Ortiz, F.; Ortiz, A.; Manana, M.; Renedo, C. (2007). Effects of voltage sags on different types of ballasts for 150-W HPS lamps. In 2007 th International Conference on Electrical Power Quality and Utilisation. IEEE, pp. 1-6. [Online] Disponible en: http://ieeexplore.ieee.org/lpdocs/epic03/ wrapper.htm?arnumber $=4424185$ [Consultado 26 de agosto de 2014].

Dorr, D.; Mansoor, A.; Morinec, A.; Worley, J. (1995). Applying HID high-pressure sodium lamps in a variablevoltage environment. In IAS '95. Conference Record of the 1995 IEEE Industry Applications Conference Thirtieth IAS Annual Meeting. IEEE, pp. 2059-2065. [Online] Disponible en: http://ieeexplore.ieee.org/ lpdocs/epic03/wrapper.htm?arnumber $=530564$ [Consultado 26 de agosto de 2014].

Fluke Corporation (2008). Fluke 434/435: Three Phase Power Quality Analyzer. [Online] Disponible en: http://assets.fluke.com/manuals/434_435_ umeng0300.pdf [Consultado 10 de julio de 2014].

International Electrotechnical Commission (2006). IEC61000-4-3 Testing and measurement techniques Radiated, radio-frequency, electromagnetic field immunity test 3rd ed., USA: Iso. [Online] Disponible en: http://www.ncbi.nlm.nih.gov/pubmed/21144490.

International Rectifier (2003). Datasheet: ST180S SERIES., p.8. [Online] Disponible en: http://www.irf. com/product-info/datasheets/data/st180s.pdf [Consultado 10 de junio 2014].

Markiewicz, H.; Klajn, A. (2004). Voltage Disturbances: Standar EN 50160- Voltage Characteristics in Public Distribution Systems. 5.4.2, pp. 1-12. [Online] Disponible en: http://admin.copperalliance.eu/docs/ librariesprovider5/power-quality-and-utilisationguide/542-standard-en-50160-voltage-characteristics-in.pdf?sfvrsn=4\&sfvrsn=4.

MOTOROLA (2005). Data Sheet MC68HC08JL3. , pp.1-3. [Online] Disponible en: http://cache.freescale.com/ files/microcontrollers/doc/data_sheet/MC68HC08JL3.pdf.

Perez Fernandez, E. (2006). Nuevo método de detección y análisis en tiempo real de eventos en la tensión de su- 
ministro de energía eléctrica empleando un modelo combinado Wavelets-filtro Kalman extendido. Tesis Doctoral. Universidad de Cantabria. Departamento de Electrónica y Computadores. 81 p.

Sedighnejad, H.; Jalilian, A. (2010). Effect of protection device coordination on voltage sag characteristics of distribution networks. ISA Transactions, 49(4), pp.407-414. [Online] Disponible en: http://www. ncbi.nlm.nih.gov/pubmed/20594552 [Consultado 26 de agosto de 2014].

Thallam, R.S.; Heydt, G.T. (2000). Power acceptability and voltage sag indices in the three phase sense. In 2000 Power Engineering Society Summer Meeting (Cat. No.00CH37134). IEEE, pp. 905-910. [Online] Disponible en: http://ieeexplore.ieee.org/xpls/abs_all. jsp?arnumber $=867482$ [Consultado 26 de agosto de 2014].

Vargas López, A.I. (2005). Subtensiones (sags \& dips) y su impacto económico en la calidad de la energía en la industria Guatemalteca. Universidad de San Carlos de Guatemala. [Online] Disponible en: https:// www.academia.edu/1832243/SUBTENSIONES_ SAGs_and_DIPs_Y_SU_IMPACTO_ECONÓMICO_EN_ LA_CALIDAD_DE_LA_ENERGÍA_E_INDUSTRIA_GUATEMALTECA.

Yim-Shu, Lee; Hon-Chee, So; Chow, M. (2009). Design of AC voltage sag compensators. In 2009 IEEE 6th International Power Electronics and Motion Control Conference. IEEE, pp. 2587-2590. [Online] Disponible en: http://ieeexplore.ieee.org/lpdocs/epic03/wrapper.htm?arnumber $=5157842$.
PARA CITAR ESTE ARTÍCULO TO REFERENCE THIS ARTICLE / PARA CITAR ESTE ARTIGO /

Restrepo Cuestas, B.J.; López Osorio, H.A. (2016). Caracterización de luminarias tipo haluro metálico ante eventos SAG. Revista EIA, 13(26), julio-diciembre, pp. 75-82. [Online]. Disponible en: https://doi.org/10.24050/reia.v13i26.850 